Draft Version April 24, 2018

Preprint typeset using $\mathrm{IAT}_{\mathrm{E}} \mathrm{X}$ style emulateapj v. 5/2/11

\title{
IMAGING THE ELUSIVE H-POOR GAS IN THE HIGH adf PLANETARY NEBULA NGC 6778
}

\author{
Jorge García-Rojas ${ }^{1,2}$ and Romano L. M. Corradi ${ }^{3,1}$ and Hektor Monteiro ${ }^{4}$ and David Jones ${ }^{1,2}$ and Pablo \\ RODRÍGueZ-GiL ${ }^{1,2}$ AND ANTONIO CABRERA-LAVERS ${ }^{3,1}$ \\ Draft version April 24, 2018
}

\begin{abstract}
We present the first direct image of the high-metallicity gas component in a planetary nebula (NGC 6778), taken with the OSIRIS Blue Tunable Filter centered on the $\mathrm{O}$ II $\lambda 4649+50 \AA$ optical recombination lines (ORLs) at the $10.4 \mathrm{~m}$ Gran Telescopio Canarias. We show that the emission of these faint $\mathrm{O}$ II ORLs is concentrated in the central parts of the planetary nebula and is not spatially coincident either with emission coming from the bright $[\mathrm{O}$ III $] \lambda 5007 \AA$ collisionally excited line (CEL) or the bright $\mathrm{H} \alpha$ recombination line. From monochromatic emission line maps taken with VIMOS at the $8.2 \mathrm{~m}$ Very Large Telescope, we find that the spatial distribution of the emission from the auroral [O III] $\lambda 4363$ line resembles that of the O II ORLs but differs from nebular [O III] $\lambda 5007$ CEL distribution, implying a temperature gradient inside the planetary nebula. The centrally peaked distribution of the $\mathrm{O}$ II emission and the differences with the [O III] and $\mathrm{H}$ I emission profiles are consistent with the presence of an H-poor gas whose origin may be linked to the binarity of the central star. However, determination of the spatial distribution of the ORLs and CELs in other PNe, and a comparison of their dynamics is needed to further constrain the geometry and ejection mechanism of the metal-rich (H-poor) component and hence, understand the origin of the abundance discrepancy problem in PNe.
\end{abstract}

Subject headings: planetary nebulae: individual — ISM: abundances — binaries: close

\section{INTRODUCTION}

In photoionized nebulae -both $\mathrm{H}$ II regions and planetary nebulae (PNe)- optical recombination lines (ORLs) provide abundance values that are systematically larger than those obtained using collisionally excited lines (CELs) (see e. g. Peimbert \& Peimbert 2006; Liu 2012, , and references therein). This is known as the abundance discrepancy problem. It is one of the major unresolved problems in nebular astrophysics, being known of for more than seventy years (Wyse 1942), and has far-reaching consequences on the measurement of abundances throughout the Universe, most often measured using CELs from ionized gas.

The abundance discrepancy factor $(a d f)$ is defined as the ratio between the abundances derived from ORLs and CELs and is usually between 1.5 and 3 (see e. g. García-Rojas \& Esteban 2007; Liu 2012), but in PNe it has a significant tail extending to much larger values. It has recently been shown that the largest abundance discrepancy factors (up to $\sim 300$ ), are reached in $\mathrm{PNe}$ with close binary central stars that have undergone a common envelope (CE) phase (Corradi et al. 2015; Jones et al. 2016). The data presented in these papers support the hypothesis that two different gas phases may coexist (Liu et al. 2006): hot, standard metallicity gas at $\sim 10000 \mathrm{~K}$ where the CELs can be efficiently excited, and much cooler $(\sim 1000 \mathrm{~K})$ gas clumps with a

\footnotetext{
${ }^{1}$ Instituto de Astrofísica de Canarias, E-38200, La Laguna, Tenerife, Spain

2 Universidad de La Laguna. Depart. de Astrofísica, E-38206, La Laguna, Tenerife, Spain

${ }^{3}$ GRANTECAN, Cuesta de San José s/n, E-38712 , Breña Baja, La Palma, Spain

${ }^{4}$ Instituto de Física e Química, Universidade Federal de Itajubá, Av. BPS 1303-Pinheirinho, 37500-903, Itajubá, Brazil
}

highly enhanced content of heavy elements (which is the cause of the cooling) where only ORLs form. As this dual nature is not predicted by mass loss theories, these results have added a new, unexpected ingredient to the abundance discrepancy problem: very high discrepancy factors should be explained in the framework of (interacting) binary evolution. Some of the proposed explanations are naturally linked to binarity, such as for instance highmetallicity nova-like ejecta (Wesson et al. 2008). Other appealing explanations involve the presence of planetary debris that survived the whole evolution of the central star, or the tidal destruction, accretion and ejection of Jupiter-like planets (see the discussion in Corradi et al. 2015), but it is difficult at this stage to favor any scenario because observational constraints are still very scarce.

The existing long-slit studies point out that the plasma emitting in O II ORLs (the brightest metal ORLs in the optical spectrum) is generally more concentrated in the central parts of the nebulae than the CEL emitting zones of the same ion (e. g. Liu 2012; Corradi et al. 2015). However, no direct imaging of the gas component where the bulk of the metal ORLs is emitted has ever been attempted, mainly because O II ORLs are faint and suitable filters are generally not available.

NGC $6778\left(\alpha=19^{h} 18^{m} 24.94^{s}, \delta=01^{\circ} 35^{\prime} 47.641^{\prime \prime}\right.$, PNG034.506.7) was found to show unusually strong ORLs reflecting a high adf value (average value $\sim 20$, see Jones et al. 2016). It hosts a close binary central star with an orbital period of 3.7 hours, making it one of the shortest period binary central stars known (Miszalski et al. 2011).

In the present work, we take advantage of the large collecting area of the $10.4 \mathrm{~m}$ Gran Telescopio Canarias (GTC) telescope and its Blue Tunable Filter (BTF) to study the $2 \mathrm{D}$ emission profiles of the $\mathrm{O}$ II ORL and [O III] 
CELs for the first time.

\section{OBSERVATIONS AND DATA REDUCTION}

\subsection{OSIRIS-GTC data}

We obtained narrow band imagery of NGC 6778 using the Optical System for Imaging and Low Resolution Integrated Spectroscopy (OSIRIS, Cepa et al. 2000, 2003) on the $10.4 \mathrm{~m}$ Gran Telescopio Canarias (GTC), at the Observatorio del Roque de los Muchachos on La Palma. The OSIRIS detector consists of a mosaic of two Marconi CCDs, each with $2048 \times 4096$ pixels and a total unvignetted field of view of $7.8 \times 7.8 \mathrm{arcmin}^{2}$, giving a plate scale of $0.127 \mathrm{arcsec} / \mathrm{pix}$. We selected the $2 \times 2$ binning mode and the $200 \mathrm{kHz}$ readout mode.

We used the OSIRIS Blue Tunable Filter (BTF) for our observations. It consists of a Fabry-Perot etalon which allows narrow band imaging at a selected wavelength. However, Fabry-Perot etalons cause a radial wavelength dependence in the form of "rings" of constant wavelength centred on the optical axis. Therefore, in order to sample the PN O II emission at $4649+50 \AA$, the BTF tuning was displaced to $465.4 \pm 0.2 \mathrm{~nm}$, with an effective bandpass of $0.85 \mathrm{~nm}$. This ensures that the wavelength selection is the desired one to isolate $\mathrm{O}$ II emission when the target is located 1 arcmin from the TF optical center. There was no need to scan the TF as the nebula is sufficiently small $(<25$ arcsec diameter excluding the faintest collimated outflows) that the wavelength variation from one side to the other of the nebula is $<3 \AA$ at the selected location.

Data for NGC 6778 were taken in service mode during the night of 2015 August 15 under Director's Discretionary Time. A total integration time of $3 \times 600$ $\mathrm{s}$ was obtained, with small offsets to remove the sky background contribution. The OSIRIS BTF images were bias- and flat-field corrected in the usual way using IRAF5. Sky emission was removed by producing an image containing only the sky emission from the series of dithered images. This sky image was then subtracted from the original images of the source, resulting in images cleaned from any background and sky emission. To save telescope time, owing to the large exposure times needed to obtain the desired $\mathrm{S} / \mathrm{N}$ ratio in the $\mathrm{O}$ II lines, no attempt was made to subtract the negligible nebular continuum.

Additionally, we show [O $\left[\begin{array}{ll}\mathrm{O} & \mathrm{III}\end{array}\right] \lambda 5007, \mathrm{H} \alpha$ and [N II] $\lambda 6548$ narrow-band images taken from Guerrero \& Miranda (2012) (see Sect. 3). All the images were registered to the [O III] image pixel scale $\left(0.189\right.$ arcsec pix $\left.^{-1}\right)$ and astrometrized using the 2MASS catalog and the script designed by Sanabria (2015).

\subsection{VIMOS-VLT data}

NGC 6778 was observed with VIMOS (Le Fèvre et al. 2015), on the ESO-VLT UT3 Melipal on the night of 2007 September 14. VIMOS is equipped with an integral field unit containing 6400 fibers and has a changeable scale on the sky that was set to 0.33 arcsec per fiber. This provided a sky coverage of $13.2 \times 13.2 \operatorname{arcsec}^{2}$ with

5 IRAF is distributed by the National Optical Astronomy Observatory, which is operated by the Association of Universities for Research in Astronomy (AURA) under a cooperative agreement with the National Science Foundation. an image formed by an array of $40 \times 40$ fibers. We obtained observations in the VIMOS high-resolution mode, providing a reciprocal dispersion of $0.6 \AA \mathrm{pix}^{-1}$ and a usable range from 3900 to $7400 \AA$ adding the blue and red grisms of the spectrograph. The object was observed with an exposure time of $300 \mathrm{~s}$ in both the red and blue configurations. The reduction was performed with the VIMOS pipelines available at the instrument website. For sky subtraction we observed fields offset by 40 arcsec. The sky level was estimated as an average for each pixel along the dispersion axis and then subtracted from the object data cube. We also applied a correction for the differential atmospheric extinction using the central star of NGC 6778 in the data cube and performing the necessary shifts along the dispersion axis. To extract the final VIMOS emission line maps we used a set of IDL routines which fit Gaussian profiles to the lines of interest in each spaxel in the data cube. In Fig. 11 we show the area covered by the VIMOS IFU by overlaying the VIMOS map taken in the light of $\mathrm{H} \alpha$ on an ALFOSCNOT narrow-band image centered on the same line from Guerrero \& Miranda (2012). The IFU was positioned to cover the central $13.2 \times 13.2 \operatorname{arcsec}^{2}$ of the nebula.

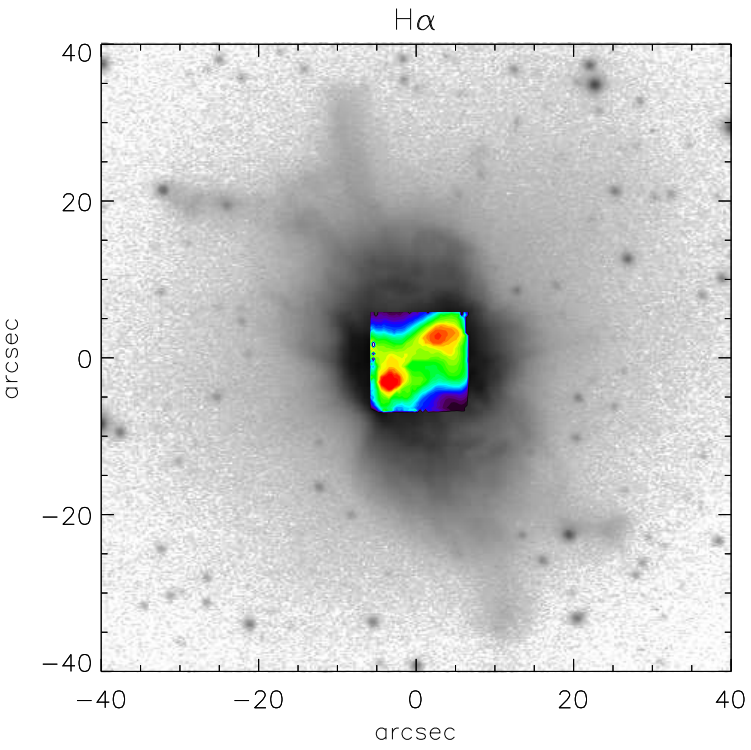

FIG. 1. - The VIMOS $\mathrm{H} \alpha$ map (color image) overlaid on ALFOSC-NOT narrow band image showing the area covered by the VIMOS IFU $\left(13.2 \times 13.2 \operatorname{arcsec}^{2}\right)$. The ALFOSC-NOT image was taken from Guerrero \& Miranda (2012).

\section{RESULTS AND DISCUSSION}

\subsection{OSIRIS-GTC and ALFOSC-NOT images}

In the upper-left panel of Fig. 2 we show the OSIRISGTC BTF image centered on the O II $\lambda \lambda 4649+51$ ORLs showing the spatial distribution of $\mathrm{O}^{2+}$ from these lines. In the upper-right panel, we show the narrowband [O III] $\lambda 5007$ CEL image from Guerrero \& Miranda (2012). The lower panels present narrow-band images in $\mathrm{H} \alpha$ (left) and [N $\mathrm{II}] \lambda 6548 \mathrm{CEL}$ (right) from the same authors. It is known that in several PNe the O II ORLs are contaminated by fluorescent $\mathrm{N}$ III lines and/or

${ }^{6}$ http://www.eso.org/sci/facilities/paranal/instruments/vimos/ 


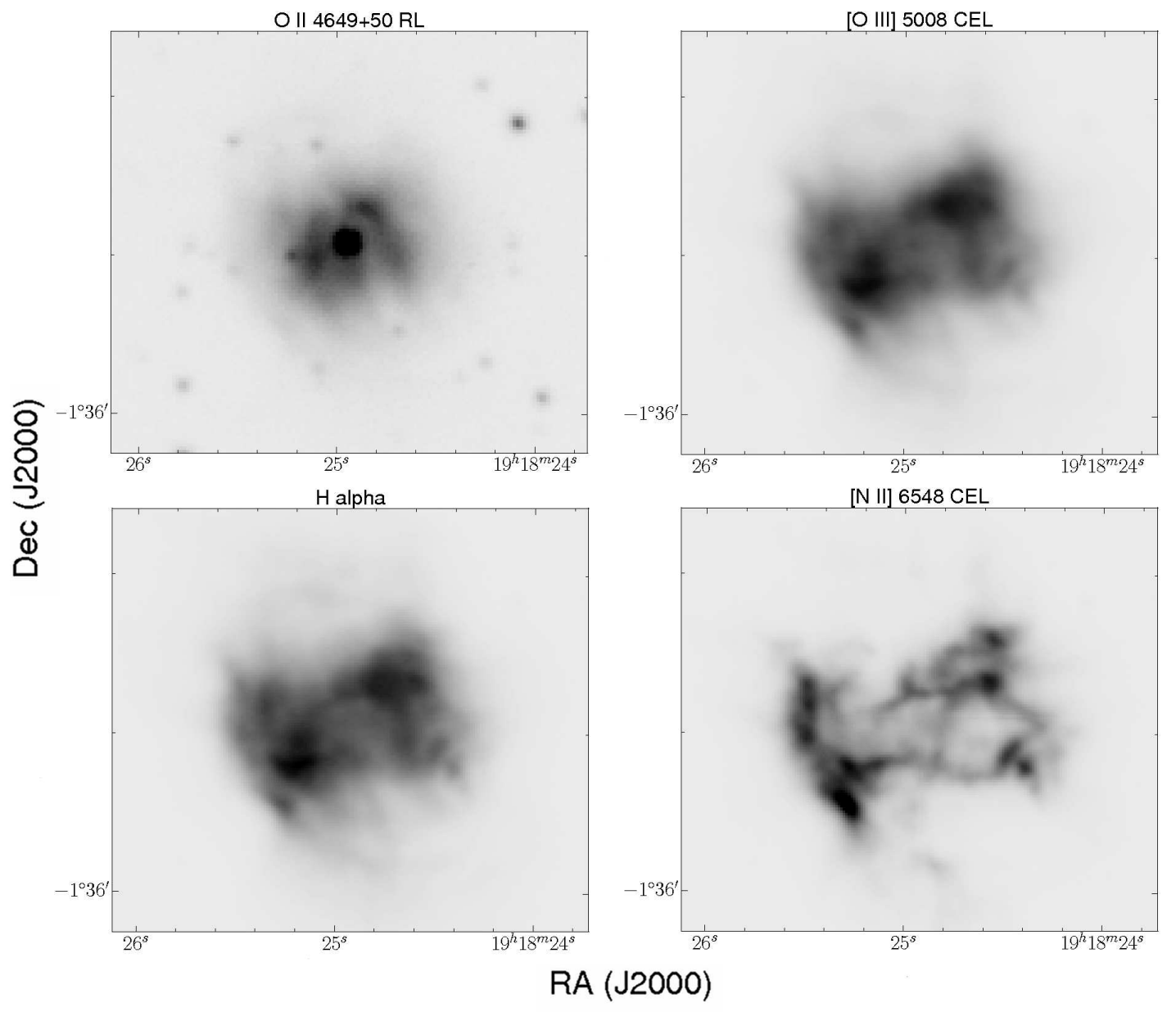

FIG. 2.- OSIRIS-GTC Tunable-filter image of NGC 6778 in the O II 4649+51 ORLs (upper left), compared to the ALFOSC-NOT images from Guerrero \& Miranda (2012) in the [O III] 5007 CEL (upper right), H $\alpha$ (lower left) and [N II] 6548 (lower right). All the images were registered to the $[\mathrm{O} \mathrm{III]} \mathrm{image} \mathrm{pixel} \mathrm{scale}(0.189 \mathrm{arcsec} / \mathrm{pixel})$ and astrometrized using the $2 \mathrm{MASS}$ catalog. It is worth mentioning that $\mathrm{O}$ II and $\left[\mathrm{O}\right.$ III] emission comes from the same ion: $\mathrm{O}^{2+}$.

$\mathrm{C}$ III ORLs. In their deep VLT-FORS2 spectrum of NGC 6778, Jones et al. (2016) did not report any contamination in the wavelength range covered by our tunable filter. In Fig. 3, we show a section of the observed spectrum of NGC 6778 around the multiplet 1 of O II. It is clear that there is no emission from the $\mathrm{C}$ III $\lambda 4647.42$ line, which is the brightest one of the multiplet 1 of C III. Therefore, all the emission covered by the tunable filter comes purely from $\mathrm{O}$ II recombination lines.

From inspection of Fig. 2, it is clear that the [O III] $\lambda 5007$ and $\mathrm{H} \alpha$ emissions share a very similar spatial distribution in the central parts of NGC 6778 (see also the discussion in Guerrero \& Miranda 2012). On the other hand, the $[\mathrm{N}$ II $] \lambda 6548$ emission has a very different spatial distribution. Guerrero \& Miranda (2012) showed that the innermost region of NGC 6778 can be described as a distorted and fragmented [N II]-bright equatorial ring aligned along a line close to the east-west direction. This [N II] CEL innermost region is characterized by the presence of filamentary low-ionization structures (LIS). Their origin, however, remains unknown but has been linked to post-CE nebulae through photoionization of neutral material deposited in the orbital plane during the CE phase (see Miszalski et al. 2011; Manick et al. 2015, and references therein).

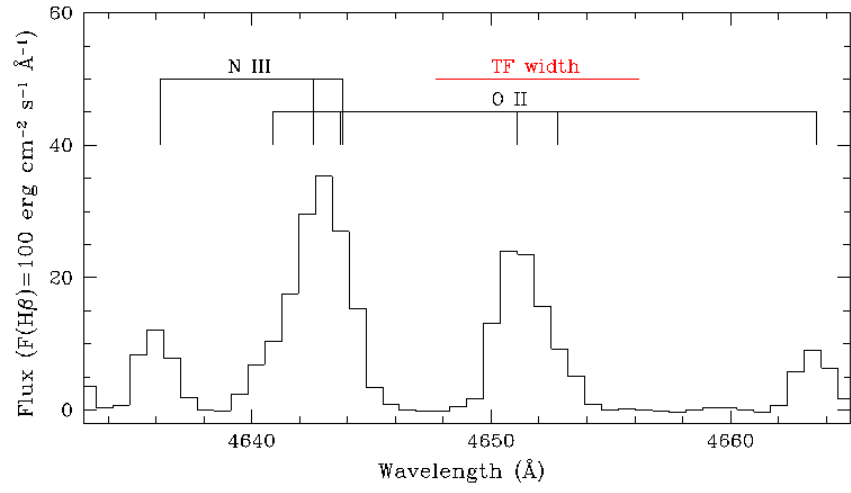

FIG. 3. - Section of the VLT-FORS2 observed spectrum (black line) of NGC 6778 presented in Jones et al. (2016) where the O II ORLs, as well as some N III lines, have been highlighted. The TF width is shown as a red line.

The most important finding from inspection of Fig. 2 is that in NGC 6778 the location of the $\mathrm{O}^{2+}$ ions producing the O II ORLs at $4649+50 \AA$ does not match the spatial distribution of the same ions emitting in the [O III] $\lambda 5007$ CEL. The O II ORL emission is concentrated inside the [O III] CEL emission or H I emission. This is the first time that the different distribution of $\mathrm{O}$ II ORL and nebular 
[O III] CEL emission has been shown using direct imaging. This finding clearly supports the hypothesis of the existence of two separate plasmas, with the additional indication that they are not well mixed, perhaps because they were produced in distinct ejection events. Previous works based on long-slit and 2D spectrophotometry in partial regions of PNe found similar results. Hence, this behaviour seems to be a standard characteristic of the ORL emission in PNe with high adfs (see e. g. Liu et al. 2000; Tsamis et al. 2008; Corradi et al. 2015).

\subsection{VIMOS monochromatic emission line maps}

We have analyzed the morphology of the emission coming from various other lines on monochromatic emission line maps taken with VIMOS-VLT. In the left column of Fig. 4, we show the C II $\lambda 4267$ and O II $\lambda \lambda 4649+50$ maps which characterize the spatial distribution of pure $\mathrm{C}^{2+}$ and $\mathrm{O}^{2+}$ recombination emission. Although the $\mathrm{C}$ II emission has a rather low contrast relative to the background, its global morphology resembles the O II $\lambda \lambda 4649+50$ emission, showing local peaks at very similar spaxels. The right column of Fig. 4 shows the resemblance of the auroral [O III $] \lambda 4363$ CEL and the nebular [O III] $\lambda 5007$ CEL emission maps. The morphologies of $\mathrm{O}$ II $\lambda 4649+50$ and [O III] $\lambda 5007$ emissions are remarkably different, and are consistent with what is found from the GTC and NOT imagery (see Fig. 1).

On the other hand, it is clear that the [O III] $\lambda 4363$ and $[\mathrm{O}$ III $] \lambda 5007$ CEL emissions have a very different spatial distribution, but [O III] $\lambda 4363$ resembles the $\mathrm{O}$ II ORL emission. This causes a radially increasing [O III $] 5007 / 4363$ flux ratio, which may be interpreted as being due to a markedly negative electron temperature gradient (Jones et al. 2016, estimated a negative gradient of more than $1000 \mathrm{~K}$, see their Fig. 5). This effect has been found in other PNe (see Liu et al. 2000; Garnett \& Dinerstein 2001, for the PNe NGC 6153 and NGC 6720, respectively). However, the presence of extremely high-density clumps $\left(n_{\mathrm{e}}>10^{5} \mathrm{~cm}^{-3}\right)$ in the inner regions would cause the quenching of nebular [O III] lines in the central part of the PN owing to collisional deexcitation effects. The effect of high-density ionized gas in the determination of chemical abundances from ORLs and CELs has been studied in detail in i. e. the Orion nebula, by Tsamis et al. (2011) and Mesa-Delgado et al. (2012). This would affect the determination of physical conditions $\left(n_{\mathrm{e}}\right.$ and $\left.T_{\mathrm{e}}\right)$ and abundances from CELs, creating a spurious temperature gradient. Moreover, if these clumps are $\mathrm{H}$-poor and only contribute a few percent of the total $\mathrm{H}$ mass of the $\mathrm{PN}$, as it has been proposed for $\mathrm{PN}$, they would not be seen in H I images. Therefore, our observations support the presence of a metal rich, dense component in the inner regions of NGC 6778, where ORL emission mainly form, in addition to the "standard", low density and H-rich gas component where CELs are emitted. Other than its location in the inner nebular regions, which may indicate a younger origin, the morphology of the ORL emitting gas does not provide any other clues to its origin. Detailed 3D photoionization models are therefore needed to fully constrain the nebula and shed further light on its formation.

All these results are in very good agreement with what was found by Jones et al. (2016), although they were limited to a 1-D portion of the nebulae as selected in their long-slit FORS2 observations.
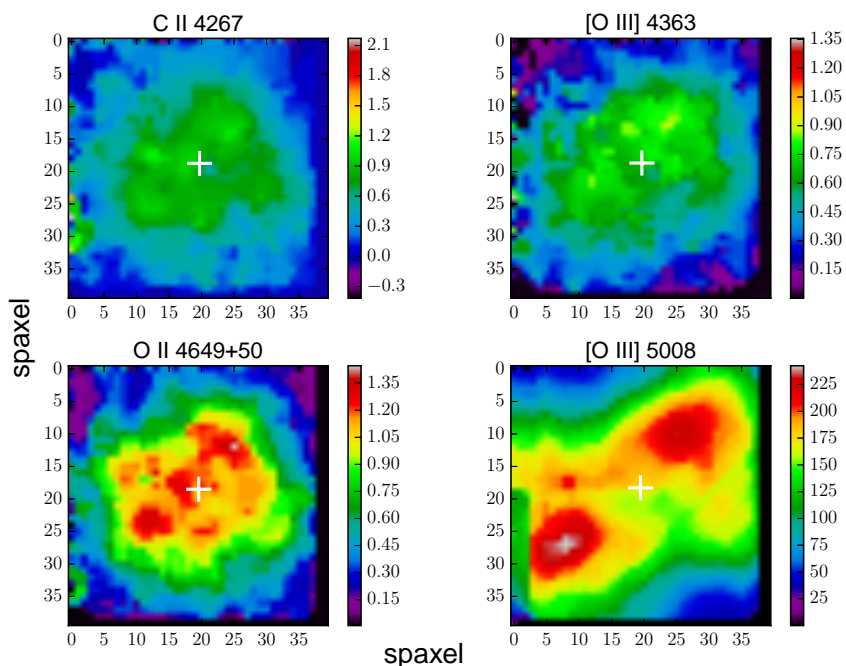

FIG. 4.- VIMOS-VLT emission line maps in several emission lines. Left column: C II $\lambda 4267$ and O II $\lambda \lambda 4649+50$ ORLs; right column: auroral [O III] $\lambda 4363$ and nebular $\lambda 5007$ CELs. The maps area is $13.2 \times 13.2 \operatorname{arcsec}^{2}$ and the orientation is north up and east left. Position of the central star is marked with a white cross.

Finally, some kinematical studies point to the presence of a colder plasma where ORLs are preferentially emitted. From very high spectral resolution observations $(\mathrm{R}=150,000)$ of two moderate to high adf $\mathrm{PNe}$ (NGC 7009 with adf $\sim 5$ and NGC 6153 with adf 10) Barlow et al. (2006) found that the full width at halfmaximum (FWHM) of $\mathrm{O}$ II ORLs were significantly smaller than that shown by [O III] CELs. They also found that auroral and nebular [O III] lines in NGC 6153 showed different velocity profiles, which implies large temperature fluctuations. Such large temperature fluctuations can also contribute to the observed adf (see García-Rojas \& Esteban 2007, and references therein). Richer et al. (2013) made a detailed kinematical study of ORLs and CELs in a limited region of PN NGC 7009. They found that ORLs exhibit different kinematics to CELs but no clear conclusion could be drawn because of the limited spatial coverage. Otsuka et al. (2010) analyzed high resolution $(\mathrm{R} \sim 33,000)$ spectra of the halo $\mathrm{PN}$ BoBn 1. They found a relatively low $a d f(\sim 2.5)$ and O II and Ne II ORLs expansion velocities lower than that of [O III] and [Ne III] CELs, which they attributed to different thermal motions. It is worth mentioning that in the case of NGC 6778, where the O II ORL emission comes from the innermost zones of the PN, the kinematics can be affected by the expansion velocity field combined with the ionization stratification of the gas in the PN. This would result in narrower O II ORLs compared to nebular [O III] CELs.

\section{CONCLUSIONS}

We present direct imaging in the light of the $\mathrm{O}$ II $\lambda \lambda 4649+50$ ORLs for the first time in a PN. We have found that the distribution of the $\mathrm{O}^{2+}$ emission coming from these lines in NGC 6778 is more centrally concentrated than the emission coming from either the strongest [O III] $\lambda 5007$ CEL or the H $\alpha$ RL. Also, from 2D reconstructed VIMOS monochromatic emission line maps, we 
find that the auroral [O III] $\lambda 4363$ CEL emission resembles the $\mathrm{O}$ II ORLs emission but not the nebular [O III] emission. This may be due to presence of a high-density, $\mathrm{H}$-poor gas component in the inner regions of the nebula, which could be responsible for the bulk of the $\mathrm{O}$ II emission and cause the abundance discrepancy. However, we do not have enough information to generalize the scenario of metal-rich inclusions to explain the bulk of low-to-moderate ADF PNe or H II regions. Moreover, the effect of high-velocity flows, such as in $\mathrm{HH}$ objects, and/or high-density inclusions, such as proplyds, has definitely something to say in the abundance discrepancy problem in $\mathrm{H}$ II regions.

The physical origin of the enhancement of the $\mathrm{O}$ II emission in the central parts of this $\mathrm{PN}$ is still elusive, but should almost certainly be linked to the binary evolution of its central star. Additional observations are needed to put this important result on solid ground and further progress in our understanding of the origin of the phenomenon. Determining the spatial distribution of the ORLs and CELs, and confronting their dynamics in several $\mathrm{PNe}$ with high $a d f$ values would help constrain the geometry and ejection mechanism of the metal rich (H-poor) component.

This work is based on observations obtained with the $10.4 \mathrm{~m}$ GTC, operating on the island of La Palma at the Spanish Observatories of the Roque de Los Muchachos of the Instituto de Astrofísica de Canarias. We thank the anonymous referee for his/her comments. This research has been supported by the Spanish Ministry of Economy and Competitiveness (MINECO) under grants AYA2012-35330, AYA2011-22614, and AYA2012-38700. JGR acknowledges support from Severo Ochoa excellence program (SEV-2011-0187) postdoctoral fellowship. PRG was supported by a Ramón y Cajal fellowship (RYC201005762). JGR acknowledges fruitful discussions with C. Esteban.

Facilities: GTC (OSIRIS) VLT (VIMOS).

\section{REFERENCES}

Barlow, M. J., Hales, A. S., Storey, P. J., et al. 2006, in IAUSymp. 234, PlanetaryNebulae in our Galaxy and Beyond, eds. M. J. Barlow \& R. H. Méndez (Cambridge: Cambridge Univ. Press), 367

Cepa J., et al., 2000, SPIE, 4008, 623

Cepa J., et al., 2003, SPIE, 4841, 1739

Corradi, R. L. M., García-Rojas, J., Jones, D., \& Rodríguez-Gil, P. 2015, ApJ, 803, 99

García-Rojas, J., \& Esteban, C., 2007, ApJ, 670, 457

Garnett, D. R., \& Dinerstein, H. L., 2001, ApJ, 558, 145

Guerrero, M. A., \& Miranda, L. F., 2012, A\&A, 539, A47

Jones, D., Wesson, R., García-Rojas, J., Corradi, R. L. M., \& Boffin, H. M. J. 2016, MNRAS, 455, 3263

Le Fèvre, O., Saisse, M., Mancini, D., et al. 2003, in SPIE Conf. Ser. 4841, eds. M. Iye ,\& A. F. M. Moorwood, 1670

Liu, X.-W. 2012, in IAU Symp. \#283, Planetary Nebulae: An Eye to the Future, Manchado, A. Stanghellini, L., \& Schönberner, D. eds., p. 131

Liu, X. -W., Storey, P. J., Barlow, M. J., Danziger, I. J., Cohen, M., \& Bryce M. 2000, MNRAS, 312, 585

Liu, X. -W., Barlow, M. J., Zhang, Y., Bastin, R. J., \& Storey P. J. 2006, MNRAS, 368, 1959
Miszalski, B., Jones, D., Rodríguez-Gil, P., Boffin, H. M. J., Corradi, R. L. M., \& Santander-García, M. 2011, A\&A, 531, A158

Mesa-Delgado, A., Núñez-Díaz, M., Esteban, C. et al. 2012, MNRAS, 426, 614

Manick, R., Miszalski, B.,\& McBride, V. 2015, MNRAS, 448, 1789

Otsuka, M., Tajitsu, A., Hyung, S., \& Izumiura, H. 2010, ApJ, 723,658

Peimbert, M., \& Peimbert, A. 2006, in IAU Symp. \#234. Barlow, M. J., \& Méndez, R. H. eds., p. 227

Richer, M. G., Georgiev, L., Arrieta, A., \& Torres-Peimbert, S. 2013, ApJ, 773, 133

Sanabria, J. J. 2015, Master Thesis, Valencian International University

Tsamis, Y. G., Walsh, J. R., Péquignot, D., et al. 2008, MNRAS, 386,22

Tsamis, Y. G., Walsh, J. R., Vílchez, J. M., \& Péquignot, D. 2011, MNRAS, 412, 1367

Wesson, R., Barlow, M. J., Liu, X.-W., et al. 2008, MNRAS, 383, 1639

Wyse, A. B. 1942, ApJ, 95, 356 